\title{
Circular Dichroism Enhancement in Large DNA Aggregates Simulated by a Generalized Oscillator Model
}

\author{
VALERY ANDRUSHCHENKO, PETR BOUŘ \\ Institute of Organic Chemistry and Biochemistry, Academy of Sciences, Flemingovo náměstí2, \\ 16610, Praha 6, Czech Republic
}

Received 14 December 2007; Accepted 26 March 2008

DOI 10.1002/jcc.21015

Published online 16 May 2008 in Wiley InterScience (www.interscience.wiley.com).

\begin{abstract}
An increased circular dichroism (CD) signal of large molecular aggregates formed upon DNA condensation was observed a long time ago, and is often referred to as $\psi$-CD. The effort to understand this phenomenon is further motivated by the latest DNA packing studies and advances in macromolecular chemistry. In the present work, the transition dipole coupling model describing interactions of molecules with light has been extended to handle systems of arbitrary size. The analytical formulae obtained retain the simplicity and computational speed of the standard approach. The origin of the $\psi$-effect was investigated on several model systems. The results suggest that the $\mathrm{CD}$ enhancement is primarily caused by delocalized phonon-like excitations in nucleic acid strands. The size of the system exhibiting the effect thus does not need to be comparable with or greater than the wavelength of the absorbed light. Small structural irregularities still allow for the enhancement while a larger disorder breaks it. The modeling is consistent with previous experimental electronic and vibrational CD studies, and makes it possible to correlate the enhancement with the geometry of the nucleic acid systems.
\end{abstract}

(C) 2008 Wiley Periodicals, Inc. J Comput Chem 29: 2693-2703, 2008

Key words: light absorption; DNA; dipolar approximation; circular dichroism; molecular aggregates; transition dipole coupling

\section{Introduction}

Spatial compacting of the DNA-encoded genetic information is necessary for its storage in the living cells. The decrease of volume occupied by the DNA molecule is usually referred to as DNA condensation. ${ }^{1,2}$ In vitro, the condensation can be achieved by various condensing agents, such as natural polyamines, cobalt hexamine, positively-charged peptides and proteins, combination of polymers and highly concentrated monovalent salts. ${ }^{1-9}$ First spectroscopic characterization of condensed DNA was done by Lerman. ${ }^{9}$ They noticed that DNA condensed by a combination of neutral polymers and a high salt concentration produces atypical electronic circular dichroism (ECD) spectral intensity of which was enhanced if compared with standard B-DNA. These unusual ECD spectra were called $\psi$-type (psi for "polymer and salt induced") circular dichroism (CD). ${ }^{9}$ Later, other types of DNA condensation, such as those achieved by multivalent metal ions or H1 histone, produced similar enhancements. ${ }^{5-17}$ It was also shown that the condensed DNA spectral enhancement is not restricted to ultraviolet light and electronic $\mathrm{CD}$, but is observable also in the infrared region for the vibrational circular dichroism (VCD). ${ }^{18}$

$\mathrm{X}$-ray and electron microscopy revealed that the condensed DNA helices are packed side to side forming thick fibers, or wrap around histone proteins. The distance between the more or less parallel-oriented helices varies between 25 and $40 \AA .{ }^{19-21}$ According to X-ray and infrared spectroscopy DNA retains the B-form secondary structure also in the condensates. ${ }^{18,19}$ The CD enhancement was thus related to a tertiary structure and explained as a consequence of the interaction of circularly polarized light with the ordered and tightly packed DNA double helices. $^{7,8}$ Alternatively, possible roles of the light scattering ${ }^{22}$ or a liquid crystalline behavior of DNA aggregates ${ }^{23}$ for the $\psi$-effect were discussed.

First consistent theory of the light interaction with large molecular aggregates based on polarizability properties of the macrosystems was successfully proposed by Keller and coworkers ${ }^{24-26}$ According to their predictions the CD enhancement can occur due to a long range coupling of the chromophores. The particle size should be about one quarter of the light wavelength, and DNA concentration within a particle high

Correspondence to: P. Bouř; e-mail: bour@uochb.cas.cz

Contract/grant sponsor: Grant Agency of the Czech Republic; contract/ grant number: 203/06/0420, 202/07/0732

Contract/grant sponsor: Grant Agency of the Academy of Sciences; contract/grant number: A400550702 
enough to enable intense three-dimensional coupling. The theory well-describes basic characteristics of the phenomenon. For example, it could resolve the discrepancy between the results of Evdokimov and others ${ }^{3,11,27}$ about presence or absence of the $\psi$-ECD in toroidal DNA condensates, by attributing it to different experimental conditions influencing the long-range coupling.

However, the polarizability approach is too general to describe fine spectral features. It cannot include individual quantum transitions except for continuous polarizability dispersion curves. Rather arbitrarily introduced transition polarizabilities realized interactions between different molecular parts. ${ }^{24-26}$ The relation of the theory to the small-size (no-enhancement) limit has not been well elaborated. Experiments where the $\psi$-effect was observed in linear DNA chains challenge the original threedimensional requirement. ${ }^{15,16}$ The electron microscopic studies suggest that the particle sizes are rather limited. Because of the variations in experimental conditions for different methods a direct relation of the sizes to the light wavelength cannot be easily established. ${ }^{11,14}$ Thus the size may not be the primary condition for the $\psi$-CD to be encountered. The enhancement is also observed in the vibrational region ${ }^{18}$ where the wavelength is much longer than for ECD.

In the present work, we further elaborate the theory describing interaction of circularly polarized light with large molecular systems, so that individual molecular transitions can be attributed to the resultant band shapes. Primarily, the Fermi golden rule $^{28}$ for one-electron process is used with the classical (nonquantized) vector potential of the light. Because of practical limitations, we apply the general theory to the coupled-oscillator approximation $^{29}$ where individual chromophores are represented by point dipoles. The oscillator model may fail for strongly coupled chromophores, such as covalently-bound molecular parts, ${ }^{30}$ but it appeared quite suitable for nucleic acids where the main light-absorbing units-the base pairs-are weakly coupled through space. ${ }^{31-34}$ Its improved version, the matrix theory, has even been successfully applied to simulations of peptide and protein spectra, where the chromophores represented by the amide groups are coupled more strongly. ${ }^{35-39}$

In the manuscript, expressions for dipolar and rotational strengths generalized for arbitrary wavelengths are derived and applied within the transition dipole coupling (TDC) model. In the next part, the conditions necessary for the CD enhancement are tested on ideal helical and toroidal dipolar systems. Finally, vibrational and electronic CD spectra for realistic DNA molecules are simulated. Input dipole moments and transitional energies are obtained from $a b$ initio computations on single AT and GC base pairs.

\section{Method}

\section{Light Absorption by Coupled Oscillators}

For one-photon processes, such as absorption and $\mathrm{CD}$, the time derivative of the transition probability between two stationary states $G$ and $E$ is given by the "Fermi golden rule" 28 as

$$
\frac{d P_{G E}}{d t} \approx \frac{2 \pi}{\hbar^{2}}\left|\left\langle G\left|V_{0}\right| E\right\rangle\right|^{2} \delta\left(\omega_{E}-\omega_{G}-\omega\right),
$$

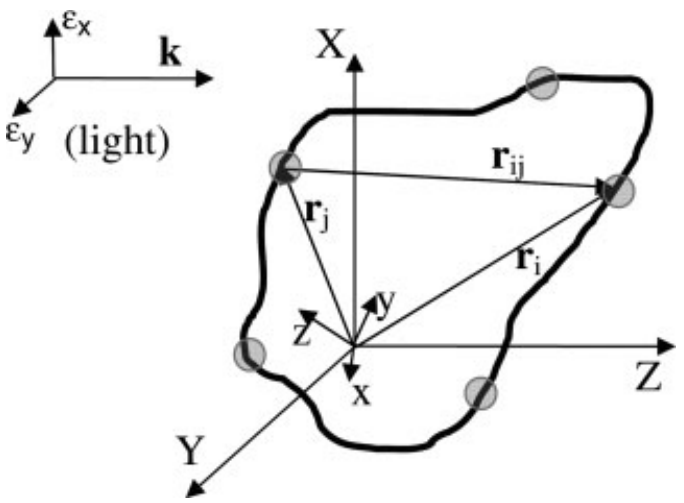

Figure 1. A light-absorbing system of independent chromophores, each one at position $\mathbf{r}_{\mathrm{i}}$. The laboratory (XYZ) and molecular ( $\left.x y z\right)$ coordinate axes are indicated. The incoming light with the wave vector $\mathbf{k}$ is propagating along the laboratory $Z$-axis, its polarization can be expressed by the unit vectors $\varepsilon_{\mathrm{X}}$ and $\varepsilon_{\mathrm{Y}}$.

where $V(t) V_{0}^{\dagger} e^{i \omega t}+V_{0} e^{-i \omega t}$ is the perturbation potential dependent on the angular frequency $\omega, h$ is the Planck constant divided by $2 \pi, \delta$ the Dirac delta-function. For a weak electromagnetic field,

$$
V=-\sum_{i} \frac{q_{i}}{m_{i}} \mathbf{p}_{i} \cdot \mathbf{A}\left(\mathbf{X}_{i}\right)
$$

where $\mathbf{A}$ is the vector potential and the sum runs over all particles in the system of respective charge, mass, momentum, and position $q_{\mathrm{i}}, m_{\mathrm{i}}, \mathbf{p}_{\mathrm{i}}$, and $\mathbf{x}_{\mathrm{i}}$. By default the SI units are used and relativistic effects are neglected. The interaction is commonly simplified by neglecting variations of the vector potential within the molecules and setting it to a constant, $\mathbf{A}\left(\mathbf{X}_{i}\right) \approx \mathbf{A}(0)$ (the dipolar approximation), or considering linear dependence on the distance only. ${ }^{28}$ This approach, however, cannot be applied to our systems, sizes of which are comparable with the light wavelength. For such cases we use a simplification utilizing division of the system into separate chromophores. The molecule is approximated by a system of $N$ oscillators of positions $\mathbf{r}_{\mathrm{i}}$, such as those in Figure 1.

Within the TDC model ${ }^{29,34}$ the ground state function is given by a product $|G\rangle=\prod \psi_{0 i}$ consisting of ground functions $\psi_{0 i}$ of individual chromophores. Similarly, the excited states can be expressed in a basis of localized excited states $\varphi_{k}=\psi_{e k} \prod \psi_{0 i}$, where $\psi_{\mathrm{ek}}$ describes an excited chromophore $k$. Also, we ${ }^{i \neq k}$ suppose that the excited energy levels are nearly degenerate. Then their coupling to the ground state can be neglected and the Schrödinger equation solved for excited states only, which yields them as a linear combination $|E\rangle=\sum_{{ }} c_{E i} \varphi_{i}$. The Hamiltonian matrix is $H_{0}+w$, where $H_{0 i j} \stackrel{i=1 \ldots N}{=} e_{i} \delta_{i j}, e_{\mathrm{i}}$ is transition energy of oscillator $i$, and the dipole-dipole interaction $w$ provides the off-diagonal $(k \neq l)$ elements

$$
W_{k l}=\left\langle\varphi_{k}|w| \varphi_{l}\right\rangle=\frac{1}{4 \pi \varepsilon_{0}}\left(\frac{r_{k l}^{2} \boldsymbol{\mu}_{k} \cdot \boldsymbol{\mu}_{l}-3 \boldsymbol{\mu}_{k} \cdot \mathbf{r}_{k l} \boldsymbol{\mu}_{l} \cdot \mathbf{r}_{k l}}{r_{k l}{ }^{5}}\right) .
$$

The transition dipoles $\boldsymbol{\mu}_{k}=\left\langle\psi_{0 k}|\boldsymbol{\mu}| \psi_{e k}\right\rangle$, their energies and positions must be input into TDC computations as parameters, 
the coefficients $c_{E i}$ are obtained by diagonalization of the Hamiltonian.

Now, we revert to the Fermi golden rule and investigate the transition matrix element for right circularly polarized light (CPL) with a potential

$$
\begin{aligned}
\mathbf{A}_{R}(\mathbf{r}, t) & =a\left(\boldsymbol{\varepsilon}_{X} \cos (k Z-\omega t)+\boldsymbol{\varepsilon}_{Y} \sin (k Z-\omega t)\right)=\frac{a}{2}\left[\left(\boldsymbol{\varepsilon}_{X}+i \boldsymbol{\varepsilon}_{Y}\right)\right. \\
& \left.\times \exp (-i k Z+i \omega t)+\left(\boldsymbol{\varepsilon}_{X}-i \boldsymbol{\varepsilon}_{Y}\right) \exp (i k Z-i \omega t)\right] .
\end{aligned}
$$

After some algebra, we obtain the quantum matrix element in Eq. (1) as

$$
\begin{aligned}
& \left\langle G\left|V_{0}\right| E\right\rangle=\frac{i}{\hbar}\left\langle\varphi_{0}\left|\sum_{i}\left[H, q_{i} \mathbf{x}_{i}\right] . \mathbf{A}_{0}\left(\mathbf{r}_{i}\right)\right| \sum_{j=1 . . N} C_{E j} \varphi_{j}\right\rangle \approx \frac{i}{\hbar} \\
& \times \sum_{j=1 . . N} C_{E j} \mathbf{A}_{0}\left(\mathbf{r}_{j}\right) \cdot e_{j} \boldsymbol{\mu}_{j}=\frac{a i}{\hbar} \sum_{j} c_{E j} e_{j}\left(\mu_{j X}-i \mu_{j Y}\right) \exp \left(i k r_{j Z}\right),
\end{aligned}
$$

where the dipole-velocity theorem $\mathbf{P}_{j}=\frac{i m_{j}}{\hbar}\left[H, \mathbf{x}_{j}\right]$ was used and the dipole operator $\boldsymbol{\mu}=\sum q_{j} \mathbf{x}_{j}$ introduced. We assumed that sizes of the individual chromophores were small enough for the vector potential to be considered constant within the chromophore and equal to $\mathbf{A}\left(\mathbf{r}_{j}\right)$, where $\mathbf{r}_{j}$ is the chromophore position (cf. Fig. 1).

This approach can be thought of as a local dipole approximation at the chromophore level. However, within the whole molecule a correct adjustment on the light phase variation is insured by the exponential factor $\exp \left(i k r_{\mathrm{iZ}}\right)$. In this approach the higher multipolar terms describing interaction of the system with the electromagnetic field are implicitly included. For example, each dipole $\boldsymbol{\mu}_{j}$ placed at position $\mathbf{r}_{j}$ provides a magnetic moment $\mathbf{m}_{j}=\frac{1}{2} \mathbf{r}_{j} \times \dot{\boldsymbol{\mu}}_{j}$ if measured at the laboratory system of coordinates. Similar origin-dependence takes place for the quadrupole, which, however, vanishes for $\mathrm{CD}$ in the current model, similarly as in the usual approach. ${ }^{40}$

For left CPL, $\boldsymbol{\varepsilon}_{y}$ would be just replaced by $-\boldsymbol{\varepsilon}_{y}$, so that the transition probabilities for the right- and left CPLs are proportional to

$$
\begin{aligned}
&\left|\left\langle G\left|V_{0}\right| E\right\rangle^{2}\right|_{R / L}=\frac{a^{2}}{\hbar^{2}} \sum_{j} \sum_{i} c_{E i} c_{E j} e_{i} e_{j}\left(\mu_{i X} \mu_{j X}+\mu_{i Y} \mu_{j Y}\right. \\
&\left. \pm i\left(\mu_{i Y} \mu_{j X}-\mu_{j Y} \mu_{i X}\right)\right) \exp \left(i k r_{i j Z}\right),
\end{aligned}
$$

where $\mathbf{r}_{i j}=\mathbf{r}_{i}-\mathbf{r}_{j}$. Based on the transition probabilities, we can develop generalized dipole (D) and rotational (R) strengths 40,41

$$
\begin{aligned}
D & =\frac{3}{2} \sum_{i} \sum_{j} c_{E i} c_{E j}\left(\mu_{i X} \mu_{j X}+\mu_{i Y} \mu_{j Y}\right) \exp \left(i k r_{i j Z}\right) \\
& =\frac{3}{2} \sum_{i} c_{E i}^{2}\left(\mu_{i X} \mu_{i X}+\mu_{i Y} \mu_{i Y}\right) \\
& +3 \sum_{i<j} c_{E i} c_{E j}\left(\mu_{i X} \mu_{j X}+\mu_{j X} \mu_{i X}\right) \cos \left(k r_{i j Z}\right) . \\
R & =\frac{3 c i}{4} \sum_{i} \sum_{j} c_{E i} c_{E j}\left(\mu_{j Y} \mu_{j X}-\mu_{i Y} \mu_{j X}\right) \exp \left(i k r_{i j Z}\right) \\
& =\frac{3 c}{2} \sum_{i<j} c_{E i} c_{E j}\left(\mu_{i X} \mu_{j Y}-\mu_{i Y} \mu_{j X}\right) \sin \left(k r_{i j Z}\right) .
\end{aligned}
$$

Note that the dipole strength can be obtained from the linear absorption index $\varepsilon=\frac{\varepsilon_{R}+\varepsilon_{L}}{2}$ as $D=9.184 \times 10^{-3} \int \varepsilon \frac{d \omega}{\omega}$. Similarly, $R=2.296 \times 10^{-3} \int^{2} \Delta \varepsilon \frac{d \omega}{\omega}$, where $\Delta \varepsilon=\varepsilon_{R}-\varepsilon_{L}$ is the CD (for $D$ and $R$ in debye, ${ }^{2}$ and $\varepsilon$ in $\mathrm{L} \mathrm{mol}^{-1} \mathrm{~cm}^{-1}$ ). ${ }^{41}$ The strengths are thus proportional to the absorption and CD peak areas of the corresponding transitions.

For an isotropic case the strengths must be averaged over all molecular orientations, e.g. by integration over the Euler angles. ${ }^{40}$ The first term in eq. (7) containing the single sum averages directly to $\sum c_{E i}{ }^{2} \mu_{i}{ }^{2}{ }^{42}$ Likewise, the remaining terms can be obtained analytically. We follow the work of Keller and Bustamante ${ }^{24}$ using the vector algebra. Formally, the space is rotated, which obviously leads to the same results as averaging over rotations of the molecule. With the aid of a rotation-independent gradient operator the spatial Z-coordinate associated with the light propagation direction (see Fig. 1) is singled out until it can be averaged analytically.

More specifically, introducing the unit wave vector $\mathbf{k}_{\mathbf{0}}=\mathbf{k} / \mathbf{k}$, polarization vectors $\varepsilon_{X}=(1,0,0)$ and $\varepsilon_{\mathrm{Y}}=(0,1,0)$ we can write the second sum in eq. (7) as $\left\langle 3 \sum_{i<j} c_{E i} c_{E j}\left(\mu_{i X} \mu_{j X}+\mu_{i Y} \mu_{j Y}\right) \cos \right.$ $\left.\left(k r_{i j z}\right)\right\rangle=\left\langle 3 \sum_{i<j} c_{E i} c_{E j} \boldsymbol{\mu}_{i} \cdot\left(\boldsymbol{\varepsilon}_{X} \boldsymbol{\varepsilon}_{X}+\boldsymbol{\varepsilon}_{Y} \boldsymbol{\varepsilon}_{Y}\right) \cdot \boldsymbol{\mu}_{j} \cos \left(\mathbf{k} \cdot \mathbf{r}_{i j}\right)\right\rangle$. Realizing the identity $\boldsymbol{\varepsilon}_{X} \boldsymbol{\varepsilon}_{X}+\boldsymbol{\varepsilon}_{Y} \boldsymbol{\varepsilon}_{Y}=\mathbf{1}-\mathbf{k}_{0} \mathbf{k}_{0}$, we obtain $\left\langle 3 \sum_{i<j}\right.$ $\left.c_{E i} c_{E j} \boldsymbol{\mu}_{i} \cdot\left(\mathbf{1}-\mathbf{k}_{0} \mathbf{k}_{0}\right) \cdot \boldsymbol{\mu}_{j} \cos \left(k \mathbf{k}_{0} \cdot \mathbf{r}_{i j}\right)\right\rangle$. The $\mathbf{k}_{\mathbf{0}}$ vector can further be replaced by a gradient operator. Additionally, scalar products of the gradient and the dipoles are rotationally-invariant, which yields $3 \sum_{i<j} c_{E i} c_{E j} \boldsymbol{\mu}_{i} \cdot\left(\mathbf{1}+k^{-2} \nabla_{i j} \nabla_{i j}\right) \cdot \boldsymbol{\mu}_{j}\left\langle\cos \left(k \mathbf{k}_{0} \cdot \mathbf{r}_{i j}\right)\right\rangle$. The averaged cosine part can be found analytically, ${ }^{24}$ providing the sum as $3 \sum_{i<j} c_{E i} c_{E j} \boldsymbol{\mu}_{i} \cdot\left(\mathbf{1}+K^{-2} \nabla_{i j} \nabla_{i j}\right) \cdot \boldsymbol{\mu}_{j} \frac{\sin \left(k r_{i j}\right)}{k r_{i j}}$, so that

$$
\begin{aligned}
\langle D\rangle=\sum_{i} c_{E i}^{2} \mu_{i}^{2}+3 \sum_{i<j} c_{E i} c_{E j} \boldsymbol{\mu}_{i} \cdot( & \left(\mathbf{1}-\mathbf{r}_{0 i j} \mathbf{r}_{0 i j}\right) j_{0}\left(k r_{i j}\right) \\
& \left.-\left(\mathbf{1}-3 \mathbf{r}_{0 i j} \mathbf{r}_{0 i j}\right) \frac{j_{1}\left(k r_{i j}\right)}{k r_{i j}}\right) \cdot \boldsymbol{\mu}_{j},
\end{aligned}
$$

where $j_{0}\left(K r_{i j}\right)=\frac{\sin \left(k r_{i j}\right)}{k r_{i j}}$ and $j_{1}\left(k r_{i j}\right)=\frac{\sin \left(k r_{i j}\right)}{k^{2} r_{i j}^{2}}-\frac{\cos \left(k r_{i j}\right)}{k r_{i j}}$ are the spherical Bessel functions of the first kind ${ }^{i j}$ and order 0 and 1 , respectively, $\nabla_{i j}=\frac{\partial}{\partial \mathbf{r}_{i j}}$. Similarly for the rotational strength (8), realizing that $\boldsymbol{\varepsilon}_{Y}=-\boldsymbol{\varepsilon}_{X} \times \mathbf{k}_{0}$ and $\boldsymbol{\varepsilon}_{X}=\boldsymbol{\varepsilon}_{Y} \times \mathbf{k}_{0}$, we obtain

$$
\begin{aligned}
\langle R\rangle & =\left\langle\frac{3 c}{2} \sum_{i<j} C_{E i} C_{E j}\left(\mu_{i X} \mu_{j Y}-\mu_{i Y} \mu_{j X}\right) \sin \left(k r_{i j Z}\right)\right\rangle \\
& =\left\langle\frac{3 c}{2} \sum_{i<j} c_{E i} c_{E j} \boldsymbol{\mu}_{i} \cdot\left(\boldsymbol{\varepsilon}_{X} \boldsymbol{\varepsilon}_{Y}-\boldsymbol{\varepsilon}_{Y} \boldsymbol{\varepsilon}_{X}\right) \cdot \boldsymbol{\mu}_{j} \sin \left(k r_{i j Z}\right)\right\rangle \\
& =-\left\langle\frac{3 c}{2} \sum_{i<j} c_{E i} c_{E j} \boldsymbol{\mu}_{i} \cdot\left(\boldsymbol{\varepsilon}_{X} \boldsymbol{\varepsilon}_{Y}+\boldsymbol{\varepsilon}_{Y} \boldsymbol{\varepsilon}_{Y}\right) \times \mathbf{k}_{0} \cdot \boldsymbol{\mu}_{j} \sin \left(k r_{i j Z}\right)\right\rangle \\
& =\operatorname{Im}\left\langle\frac{3 c}{2} \sum_{i<j} c_{E i} c_{E j} \boldsymbol{\mu}_{i} \cdot\left(1-\mathbf{k}_{0} \mathbf{k}_{0}\right) \times \mathbf{k}_{0} \cdot \boldsymbol{\mu}_{j} e^{-k k_{0} \cdot \mathbf{r}_{i j}}\right\rangle \\
& =\frac{3 c}{2} \operatorname{Im} \sum_{i<j} c_{E i} c_{E j} \boldsymbol{\mu}_{i} \cdot\left(\mathbf{1}+k^{-2} \nabla_{i j} \nabla_{i j}\right) \times i k^{-1} \nabla_{i j} \cdot \boldsymbol{\mu}_{j}\left\langle e^{-i \mathbf{k} \cdot \mathbf{r}_{i j}}\right\rangle \\
& =\frac{3 c}{2} \sum_{i<j} c_{E i} c_{E j} \boldsymbol{\mu}_{i} \cdot\left(\mathbf{1}+k^{-2} \nabla_{i j} \nabla_{i j}\right) \times k^{-1} \nabla_{i j} \cdot \boldsymbol{\mu}_{j} \frac{\sin \left(k r_{i j}\right)}{k r_{i j}} \\
& =-\frac{3 c}{2} \sum_{i<j} c_{E i} c_{E j} \boldsymbol{\mu}_{i} \cdot \mathbf{r}_{i j} \times \boldsymbol{\mu}_{j} \frac{j_{1}\left(k r_{i j}\right)}{r_{i j}} .
\end{aligned}
$$



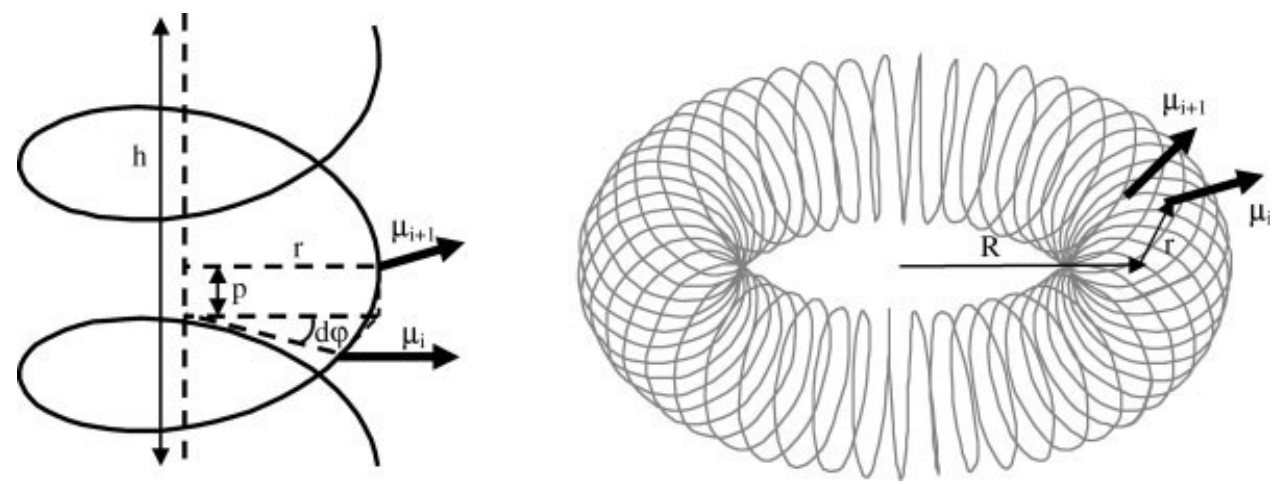

Figure 2. Geometries of the idealized dipole systems: helix (left, $p=3 \AA, r=1 \AA, d \varphi=26^{\circ}$ ) and the toroid (right).

Note that the results are independent of the choice of the coordinate origin. For long wavelengths $j_{0}\left(k r_{i j}\right) \approx 1$ and $\frac{j_{1}\left(k r_{i j}\right)}{k r_{i j}} \approx \frac{1}{3}$, and from eq. (9) we get

$$
\langle D\rangle \approx \sum_{i} C_{E i}^{2} \mu_{i}^{2}+2 \sum_{i<j} c_{E i} c_{E j} \boldsymbol{\mu}_{i} \boldsymbol{\mu}_{j},
$$

which is the usual TDC dipole strength. ${ }^{41,43}$ Likewise, eq. (10) provides the common rotational strength at the long-wavelengths limit $^{43}$ :

$$
\langle R\rangle \approx-\frac{c k}{2} \sum_{i<j} c_{E i} c_{E j} \mathbf{r}_{i j}, \cdot \boldsymbol{\mu}_{j} \times \boldsymbol{\mu}_{i}
$$

\section{Applications to Helical Systems}

Ideal helix and toroid dipolar arrangements depicted in Figure 2 were used to explore the basic behavior of the large chromophore systems. Apart from its length $h$ the helix was characterized by a rise $p$ between neighboring dipoles, an angle increment $d \varphi$, and radius $r$. Default values were set to $p=3 \AA, d \varphi$ $=26^{\circ}, r=1 \AA$; their limited variation in control calculations did not bring any qualitative change of the results. Only dipoles perpendicular to the helical axis were considered. For trial computations scalar spatial $(\Delta r)$ and dipolar $(\Delta \mu)$ dispersions were introduced, so that the dipoles $\mu_{\mathrm{i}}$ and their positions $\mathbf{r}_{\mathrm{i}}$ corresponding to the ideal arrangement were changed to $\left(\mu_{\mathrm{i}}+\Delta \mu \mathbf{e}\right)$ and $\left(\boldsymbol{r}_{\mathrm{i}}+\Delta r \mathbf{e}\right)$, where $\mathbf{e}$ is a random vector, different for each dipole and position, $|\mathbf{e}|<1$. The toroid (right hand part of Fig. 2) was constructed similarly as the helix, and characterized by the main $(R)$ and tube $(r)$ radii. The toroid dipoles were tilted towards the helical (tube) axis by $10^{\circ}$; this particular direction was chosen randomly and did not exhibit too different behavior from other possibilities not reported in the present article. Rest of the parameters $\left(p=3 \AA, d \varphi=26^{\circ}\right.$ ) for the toroidal helix were the same as for the straight helix. Transition dipole moments of $1 \mathrm{D}$ were used with a transition frequency of $33,333 \mathrm{~cm}^{-1}$ corresponding to $300 \mathrm{~nm}$ light wavelength. Longer and shorter lengths were applied in control computations.

To investigate more realistic systems, vibrational absorption and VCD spectra were simulated for a short hexanucleotide, the supercoiled DNA in a nucleosome core particle and a regular B- form $\mathrm{d}(\mathrm{CG})_{72} * \mathrm{~d}(\mathrm{GC})_{72}$ DNA helix (see Fig. 3). The short nucleotide was randomly selected from the nucleosome DNA sequence. All vibrational transition dipoles for transitions within the $1500-2000 \mathrm{~cm}^{-1}$ frequency range were transferred to each CG and AT pair from previous computations. ${ }^{44}$ The dipoles were calculated within the harmonic approximation with the Gaussian software ${ }^{45}$ at the BPW $91^{46} / 6-31 G^{* *}$ level for planar Watson-Crick base pairs. Contributions of other molecular parts to the spectra were neglected. Similarly, by Gaussian, the electronic transition dipoles and energies were calculated for the AT and $\mathrm{CG}$ pairs, at the $\mathrm{B} 3 \mathrm{LYP}^{47} / 6-31++\mathrm{G}^{* *}$ level of approximation within the time dependent density functional theory (TD DFT), ${ }^{48,49}$ and transferred to DNA.

For all dipolar systems, the TDC Hamiltonian was diagonalized, which provided the transition energies and excited wavefunctions. The dipole and rotatory strength were computed utilizing eqs. (9)-(10). The actual spectra were plotted with Lorentzian band shapes assigned to each transition, with a default full width at half maximum of $20 \mathrm{~cm}^{-1}$.

\section{Results and Discussion}

We have performed multiple numerical tests comprising simulations of both electronic (ECD) and vibrational (VCD) circular dichroism of extensive model systems; only the most characteristic results illustrating the nature of the enhancement are reported. For the ideal helix, for example, the dependence of the absorption and CD spectra on the number of dipoles was modeled within the $1 . .1000$ interval. Spectra for 1, 2, 10, 20, 50, 100, and 1000 dipoles are plotted in Figure 4 as normalized to one chromophore. The absorption can be seen in the bottom panel of the figure. The peak of the isolated oscillator $(n=1$, black line) splits for $n=2$ (red line) into two peaks corresponding to "in phase" and "out of phase" coupling, as it is common in the TDC scheme. ${ }^{29,30,34}$ The original $n=1$ band is in the middle between the smaller out of phase and a more intense in phase band of the dimer. As expected, the out of phase and in phase CD signals (top panel of Fig. 4) are exactly the same in magnitude but opposite in sign.

For longer helices $(n=10 . .1000)$, however, only the "in phase" absorption intensity is conserved and quickly saturates as 
the length increases. The absorption maximum for $n=100$ is only $\sim 10 \%$ smaller than that for $n=1000$. The peak position shifts to higher wavenumbers, but stabilizes at $\sim 33,630 \mathrm{~cm}^{-1}$. It is also important to note that for bandwidths corresponding to the experimental band broadening the absorption spectra would be independent of the helical length, because the integrated absorption intensity is always constant within the TDC model. The frequency difference between the absorption maxima of an infinite helix and the monomer $(n=1)$ is twice as large as that between $n=1$ and 2. The CD intensity builds up at the same frequency as the absorption. However, the CD signal is saturated much later than the absorption. Compared with the longest helix ( $n=1000$ ), $n=10$ provides $60 \%$ of the absorption height, but only $\sim 15 \%$ of the CD signal, etc. Compared with $n=2$, the $\Delta \varepsilon / \varepsilon$ ratio can be increased about 50 -fold. The simulated lengthdependence of the CD spectra is thus in qualitative and quantitative agreement with the many-fold increase observed for experimental $\psi-\mathrm{CD},,^{9-16}$ even when the enhancement is not related to the dimer, but to an average straight DNA segment. Also the simulated slight frequency shift (e.g., $n=2$ vs. $n=1000$ ) is often observed in experimental $\psi$-CD spectra.

Even though the system size becomes comparable with the light wavelength for $n \sim 1000$, this does not appear to be a necessary condition for the enhancement. Indeed, for the default width $\left(h=20 \mathrm{~cm}^{-1}\right)$ the $\mathrm{CD}$ intensity enhancement grows most steeply for $n=\sim 10 \ldots .50(\Delta \varepsilon / \varepsilon$, see the inset graph in Fig. 4), which corresponds to rather short helical lengths of 3$15 \mathrm{~nm}$. For these lengths, the generalized $(9,10)$ and classical $(11,12)$ formulae for the dipole and rotatory strengths provide virtually the same spectral shapes for the bandwidth used. However, the intensity pattern would be different for highlyresolved spectra. This can be seen in Figure 5 where for $n=$ 1000 the dipole and rotational strengths are plotted for individual transitions in the vicinity of the most intense signal. The exact formulae 9-10 provide a significantly different intensity distribution between individual transitions than the classical limit, while the total zero (peak area, for the absorption) and first (for CD) spectral moments are conserved, in accordance with the general sum rules. ${ }^{40}$ In other words, the detailed intensity distribution is affected by the phase variation of the light vector potential (electric intensity) sampling the excited wavefunctions delocalized at different positions in the system.

Larger bandwidths produce spectra of similar shapes, but the enhancement diminishes. For a constant splitting and bandshape, the ratio of the $\mathrm{CD}$ and absorption intensity is inversely proportional to the width. ${ }^{50}$ This can be seen at the inset of Figure 4 for the width of $100 \mathrm{~cm}^{-1}$. Larger widths thus significantly damp the enhancement, but might not destroy it completely. This is in agreement with modest enhancements observed in the UV region, often of the order of tens of percents only. ${ }^{15}$ A typical experimental CD $\Delta \varepsilon / \varepsilon$ ratio of a B-form DNA is about $10^{-3}-10^{-4}$. The value can increase about 10 times in conditions most favorable to the $\psi$-effect. ${ }^{12,15,27}$ This indicates a presence of many factors destroying the ideal limit simulated in Figure 4. On the other hand, larger band splitting in real systems may lead to an increase of the enhancement and balance the width modulation, for example. Particularly, the dipolar interaction (3) proportional to the square of the dipole moment can sharply rise for strong dipolar transitions.

In Figure 6, left, structural irregularities in real systems are simulated by random perturbations of the transitional dipole moments. It is apparent that this decreases the enhancement, too. For the absorption, the signal becomes broader; the peak area is nevertheless conserved. On the contrary, for large perturbations, the CD signal almost vanishes because of many contributions of different signs. Still, for mild irregularities (e.g., spectra $b, b^{\prime}$ in Fig. 6), a significant enhancement remains.

Under constant dipoles and geometry, variation of the diagonal matrix elements, the unperturbed frequencies, also damps the enhancement effect, as can be seen from the dependence of the $\Delta \varepsilon / \varepsilon$ ratio on the dispersion plotted in the right hand side of Figure 6 . Random perturbations limited by $\Delta \omega$ were added to the frequencies. Significant decrease of the $\mathrm{CD}$ enhancement can be seen; for example, the $\Delta \varepsilon / \varepsilon$ reduces by half for $\Delta \omega=0.4 \%$ $\sim 1.2 \mathrm{~nm}$. Such a frequency variation can easily be induced in the chromophores by an inhomogeneous environment. On the other hand, for the highly-organized regular structures generally assumed for the DNA systems exhibiting the $\psi$-effect the transitional frequencies may be quite uniform, in favor of the $\psi$ effect. Overall, we can thus conclude that although many factors contribute to the CD intensity of the DNA assemblies, the longrange excitonic coupling in longer molecular segments still appears as the most probable mechanism of the enhancement.

The enhanced CD DNA spectra are thus explicable by an increased rigidity and regularity of the DNA helices in the $\psi$ systems, rather than by their large dimensions. Most likely, the arrangement of DNA double helices into super-helical threads $^{11,14}$ or other regular arrangements provide the necessary rigidity. Experimentally, a decrease of the VCD signal due to a disturbance of the regular double stranded DNA helix was observed upon interaction with various agents. ${ }^{51,52}$ At normal physiological conditions DNA exists as a worm-like chain with rather short straight segments, not favorable for the enhancement. It is the presence of various condensing agents that allows the DNA helices to arrange more regularly.

The toroidal dipole arrangement, even though regular, does not always lead to substantial enhancement of the CD spectra. This is demonstrated in Figure 7, where the absorption and CD spectra are simulated for a thick (left) and a thinner (right) toroid $(r=10$ and $1 \AA$, respectively). For the former, absorption and $C D$ spectral patterns quickly stabilize as the number of the dipoles (toroid main radius $R$ ) increases. The spectral shapes appear somewhat more complicated than for the ideal straight helix. Most importantly, however, the CD intensity (see the $\Delta \varepsilon$ scale) remains quite low if compared with the straight helix (e.g. those in Fig. 4). On the other hand, the spectra of the thin tube (right-hand side of Fig. 7) exhibit similar size-dependence as the helix, particularly the huge CD intensity observed in larger toroids. Thus, the dipolar coupling favorable to the CD enhancement can be destroyed by the changes of the toroid geometry. The exact coupling magnitude between neighboring dipoles is thus also important for the enhancement.

The behavior of the idealized systems is also reflected in the vibrational absorption and VCD spectra of the actual DNA molecules structures of which are shown in Figure 3. The toroidal 


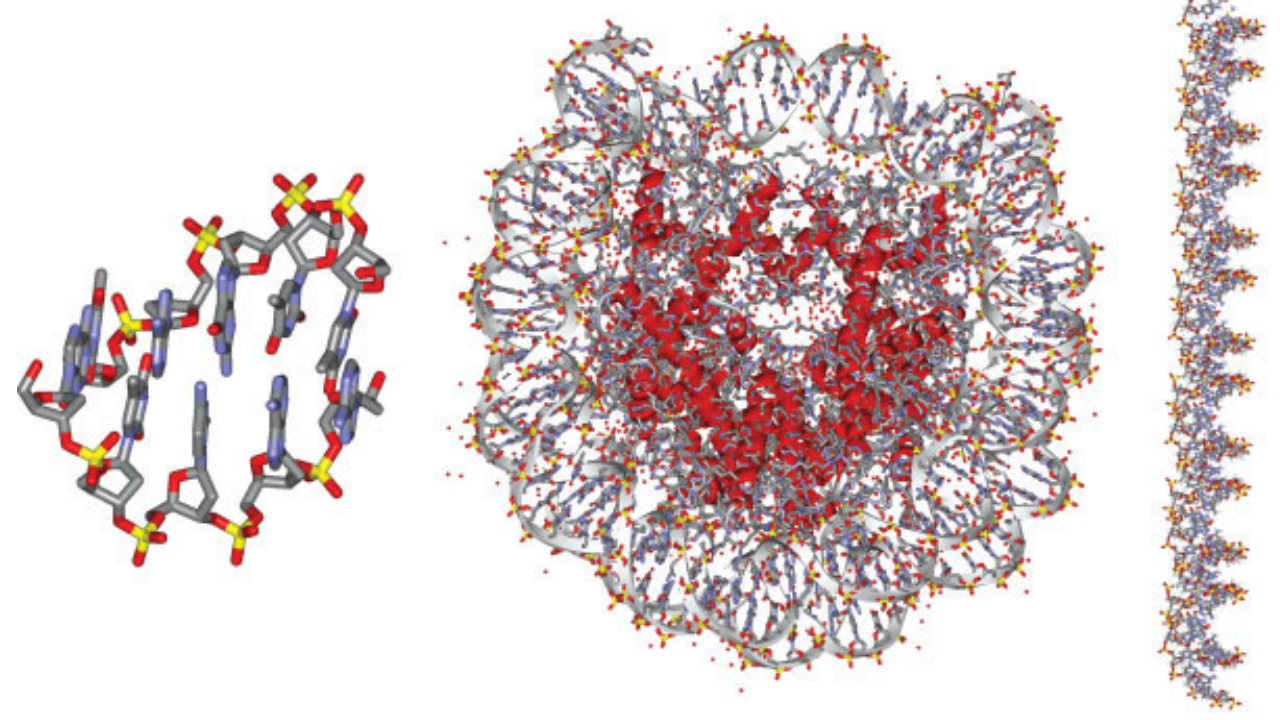

Figure 3. The DNA model molecules: (left) short AAACA segment, (middle) supercoiled DNA in the nucleosome core particle NCP146 ${ }^{20}$ containing 146 CG and AT pairs, and (right) idealized B-conformation $\mathrm{d}(\mathrm{CG})_{72} * \mathrm{~d}(\mathrm{GC})_{72}$ helix.

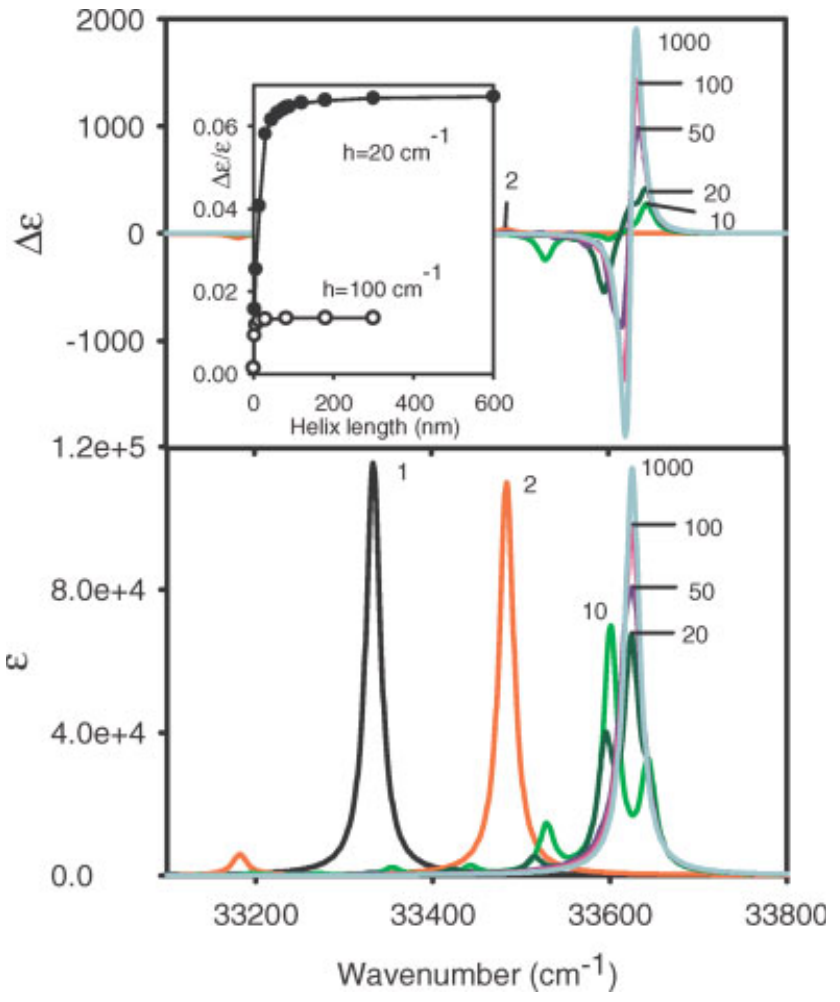

Figure 4. Calculated $\mathrm{CD}$ and absorption spectra of the ideal helix (see Fig. 2) for 1, 2, 10, 20, 50, 100, and 1000 dipoles. The inset graph shows the maximum $\Delta \varepsilon / \varepsilon$ ratio as a function of the helix length for the default and larger band width $(h=20$ and 100 $\left.\mathrm{cm}^{-1}\right)$.

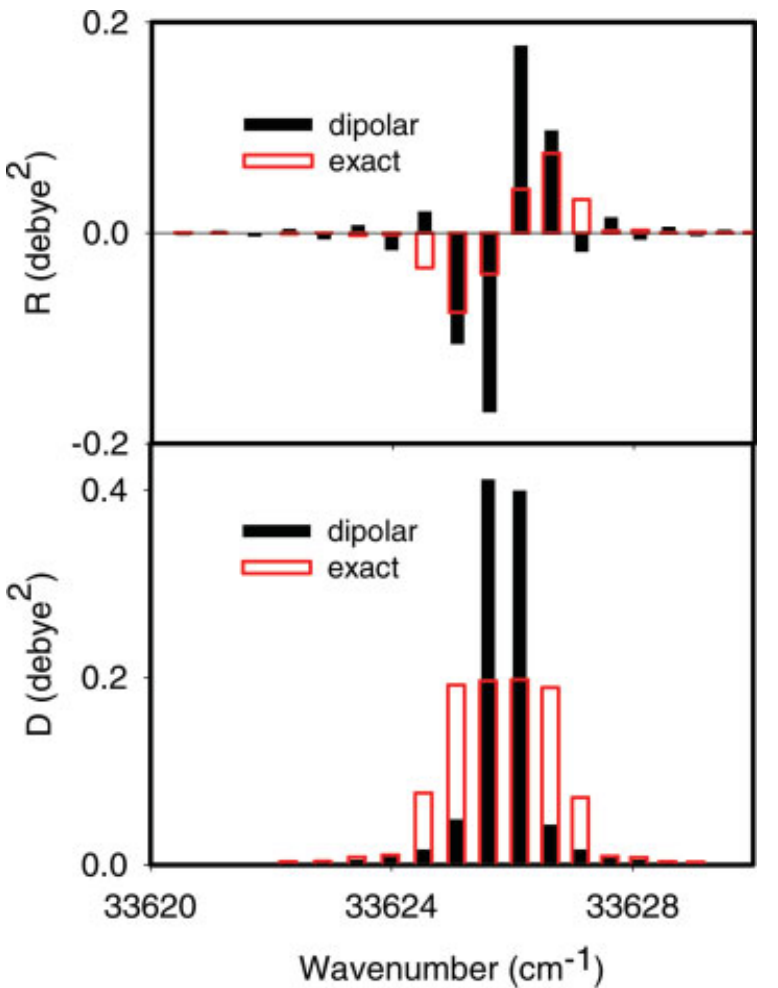

Figure 5. Patterns of rotational $(R)$ and dipolar $(D)$ strengths obtained for an ideal helix 300-nm long by the generalized formulae [eqs. (9) and (10)], and by the long-wavelength approximation [eqs. (11) and (12)]. 


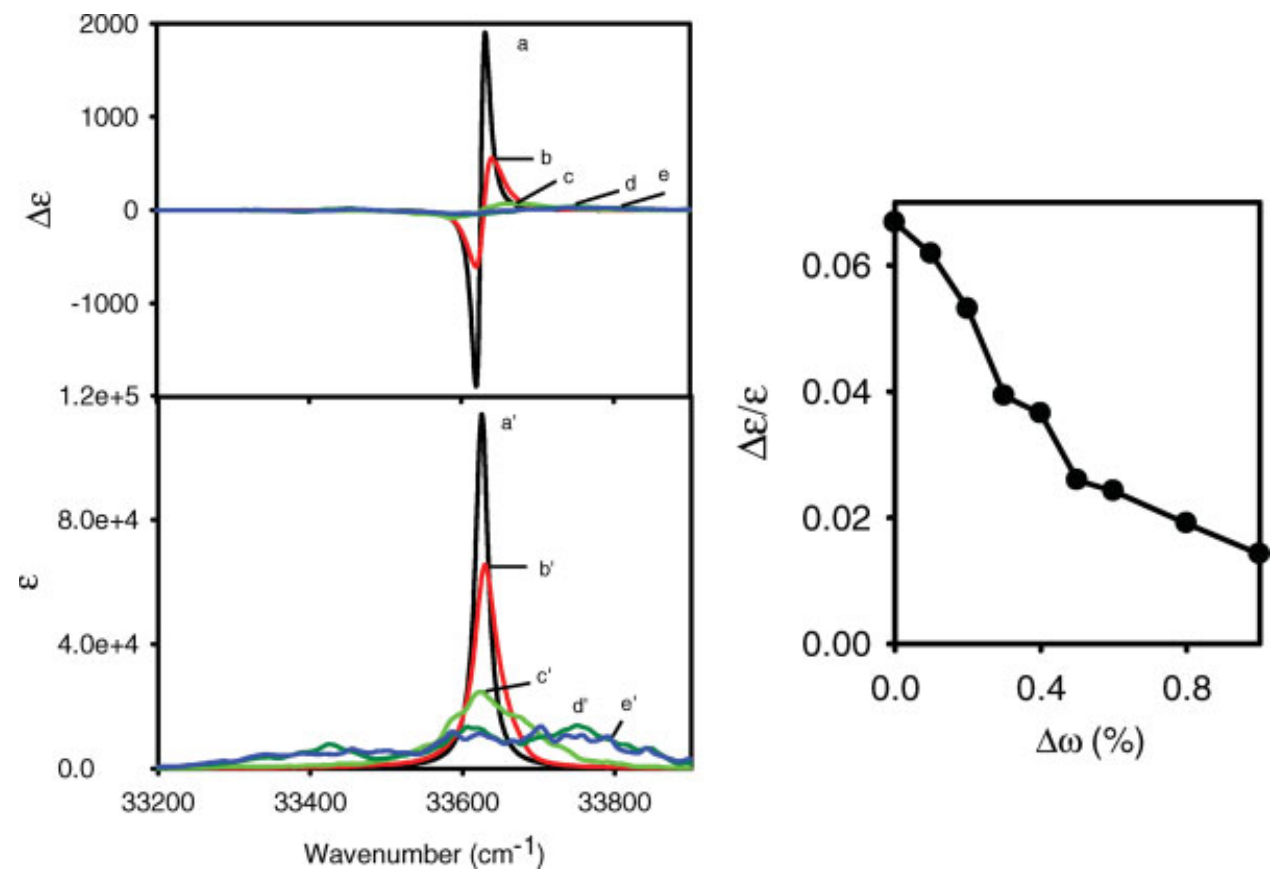

Figure 6. Left: $\mathrm{CD}(\mathrm{a}-\mathrm{e})$ and absorption $\left(\mathrm{a}^{\prime}-\mathrm{e}^{\prime}\right)$ spectra of 1000 dipoles arranged in a perfect helix (a, $\mathrm{a}^{\prime}$ ), with random maximum distance perturbations of $0.5 \AA\left(\mathrm{b}, \mathrm{b}^{\prime}\right)$ and $1 \AA\left(\mathrm{c}, \mathrm{c}^{\prime}\right)$, with a random dipole perturbation of 0.5 debye $\left(\mathrm{d}, \mathrm{d}^{\prime}\right)$ and combined dipole and distance perturbations $(0.5$ debye, 0.5 $\AA$, e, $\mathrm{e}^{\prime}$ ). Right: Dependence of the $\Delta \varepsilon / \varepsilon$ ratio on a random frequency dispersion for unchanged dipoles and distances.

superhelical nucleic acid in nucleosome particle provides a rather small VCD signal (middle of Fig. 8) with $\Delta \varepsilon / \varepsilon \sim 5 \times$ $10^{-5}$, which is about the typical VCD ratio found for DNA spectra experimentally. ${ }^{18}$ Still, this value is higher than for the short nucleotide sequence (left-hand side of the Fig. 8). This suggests, that the DNA tertiary arrangement in the nucleosome
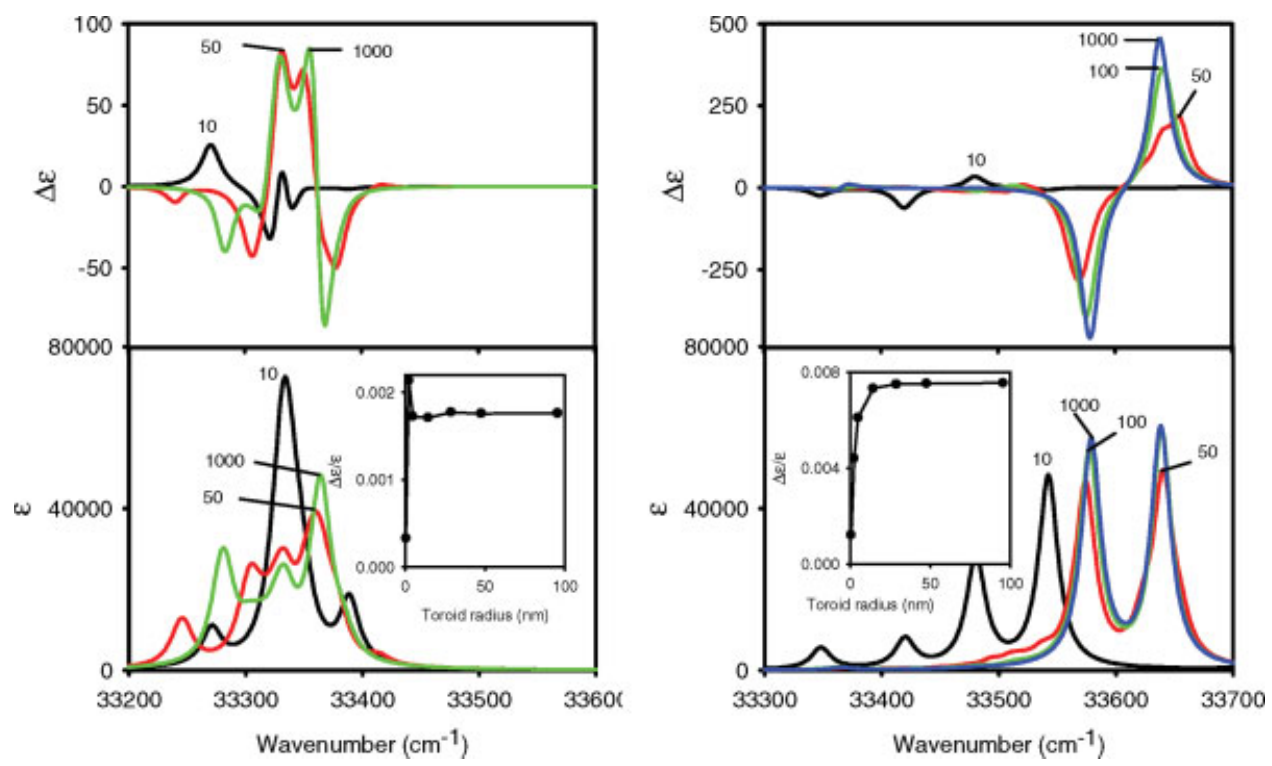

Figure 7. Calculated CD and UV absorption spectra of the toroid (see Fig. 2) with indicated number of dipoles. The inset graph shows the maximum $\Delta \varepsilon / \varepsilon$ ratio as a function of the toroid radius $R$ (see Fig. 2). Left: thick toroid ( $r=10 \AA$, no enhancement), right: thin toroid tube ( $r=1 \AA$, exhibiting CD enhancement). 

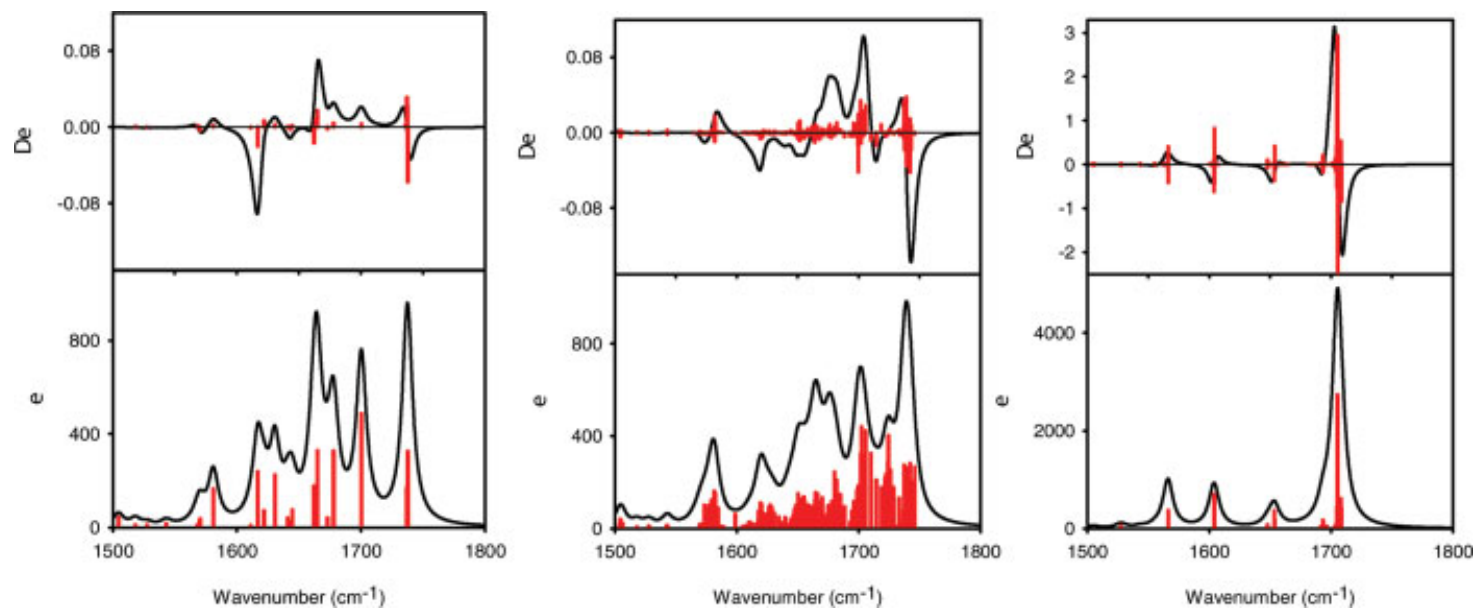

Figure 8. VCD (top) and IR absorption (bottom) spectra simulated for the 6-nucleotide (left), nucleosome (middle), and the regular (right) DNA molecules shown in Figure 3. Transition vibrational electric dipole moments were obtained by the BPW91/6-31G** computation and transferred on the DNA base pairs. The protein part of the nucleosome was not used in the calculations. [Color figure can be viewed in the online issue, which is available at www.interscience.wiley.com.]

favors a weak $\psi$-effect limited by the structural irregularities and a small particle size. On the other hand, the regular $\mathrm{d}(\mathrm{CG})_{72} * \mathrm{~d}(\mathrm{GC})_{72}$ polymer provides a large VCD signal (righthand side of Fig. 8) and the $\Delta \varepsilon / \varepsilon$ ratio of $\sim 7 \times 10^{-4}$ is rather impressive for the vibrational domain. Also note, that the $+-\mathrm{VCD}$ spectral pattern at $1700 \mathrm{~cm}^{-1}$, typical for normal BDNA, is preserved in our simulation. This pattern, however, is conserved only in some experimental $\psi$-spectra. ${ }^{18}$

However, somewhat different behavior of the short B-DNA helix, nucleosome DNA, and the regular $\mathrm{d}(\mathrm{CG})_{72} * \mathrm{~d}(\mathrm{GC})_{72}$ polymer can be observed for the ECD spectra simulated in Figure 9. Here, the short helix provides a relatively larger signal than for
VCD. The nucleosome ECD is the smallest, similarly as its VCD, which can in both cases be explained by an irregular arrangement of the chromophores due to DNA wound around the proteins, which is unfavorable to long-range exciton modes. The regular $\mathrm{d}(\mathrm{CG})_{72} * \mathrm{~d}(\mathrm{CG})_{72}$ polymer (right-hand part of Fig. 9) does provide an enhancement which is nevertheless much smaller if compared with VCD. This confirms that the enhancement is also dependent on the fine dipolar coupling pattern. The vibrational and electronic transition dipoles have different directions and magnitudes. In the experimentally most accessible frequency region of $\sim 1000-2000 \mathrm{~cm}^{-1}$ the vibrational dipoles are oriented parallel to the base pair planes. In usual electronic spec-
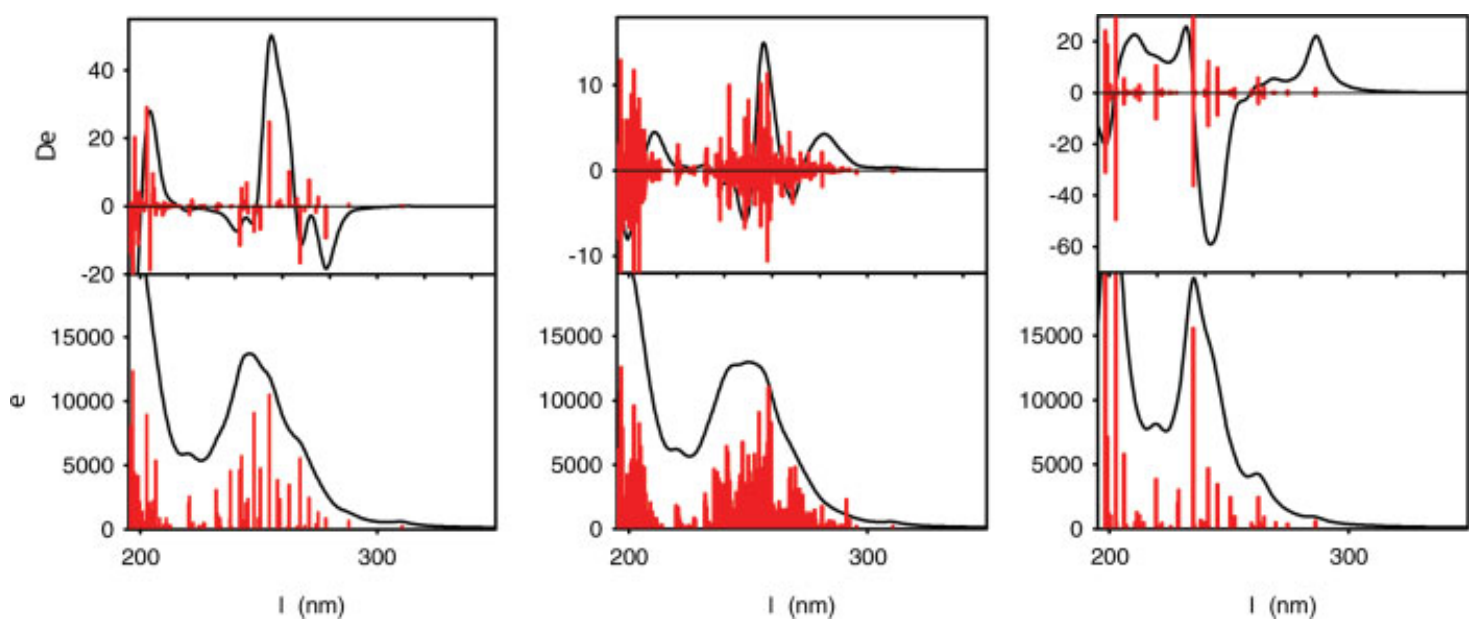

Figure 9. ECD (top) and UV absorption (bottom) spectra simulated for the 6-nucleotide (left), nucleosome (middle), and the regular (right) DNA molecules from Figure 3. Similarly as for Figure 8, the transition electric dipole moments were obtained by the TD DFT/B3LYP/6-31++G** for the DNA base pairs only. [Color figure can be viewed in the online issue, which is available at www.interscience. wiley.com.] 


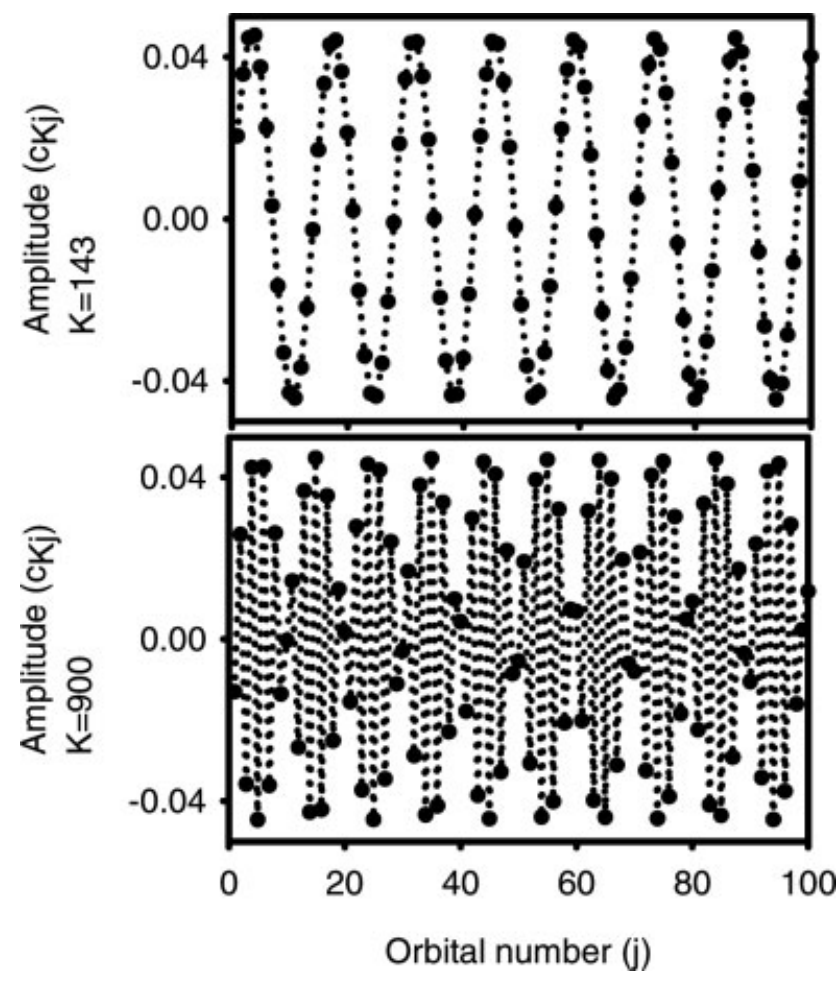

Figure 10. Example of orbital coefficients for the ideal helix: (top) phonon state number 143 with the highest absorption and CD intensity, and (bottom) excited state number 900 yielding low spectral signals.

tra (with wavelengths of $\sim 190-400 \mathrm{~nm}$ ) perpendicular transition electronic dipoles also occur, but are mostly weaker than the inplane moments. However, other tests within the present theoretical model did not reveal substantial qualitative differences between the ECD and VCD $\psi$-effects.

The simulations bring about a conclusion that unperturbed regular large DNA segments are necessary for the CD enhancement, so that long-range phonon-like excitations can develop. Indeed, if we plot the amplitudes $c_{\mathrm{Ej}}$ for the ideal helix, for example $(n=1000$, only 100 coefficients are plotted in Figure 10), we find a striking difference between the modes providing maximum absorption and CD intensity (mode number 143 is plotted in the top part of Fig. 10) and the low-intensity modes, such as number 900 plotted in the bottom part. The intense mode coefficients follow a coherent sinusoidal pattern, while for the other modes, although some regularities stemming from the helix symmetry are still apparent, the amplitude oscillations appear more erratic.

The phonon-like character of the modes can be deduced considering a standing wave solution, $\psi_{K}=A_{K} \sum_{j} e^{i f_{K j} j} \varphi_{j}$ $+B_{K} \sum_{j} e^{-i f_{K j} j} \varphi_{i}=\sum_{j} C_{K j} \varphi_{j}$. From the orthonormality condition, we get $\sum C_{K j}{ }^{*} C_{L j}=\delta_{K L}$. For many degenerate oscillators and after retaining the nearest neighbor interactions only, we can write the Hamiltonian matrix elements as $H_{j j^{\prime}}=\varepsilon \delta_{j j^{\prime}}+$ $V\left(\delta_{j+1 . j^{\prime}}+\delta_{j-1 . j^{\prime}}\right)$. The integral is then $\langle K|H| L\rangle=\varepsilon \delta_{K L}+V \sum_{j}$ $\left(C_{K j+1}{ }^{*}+C_{K j-1}{ }^{*}\right) C_{L j}=\left(\varepsilon+2 V \cos \left(f_{K}\right)\right) \delta_{K L}$. Thus the standing waves conform the Schrödinger equation for the TDC Hamiltonian. Moreover, the amplitude of the coherent state $(K=143)$ oscillates relatively slow (see Fig. 10), so that $f_{\mathrm{K}}=0$ and $\cos \left(f_{K}\right) \sim 1$. The band frequency is then equal to $\varepsilon+2 V$. The $2 V$ frequency shift can be identified in Figure 4 as the frequency separation of the long helices' signal from that of the monomer. The dimer, in contrast, exhibits two split bands with energies $\varepsilon$ $\pm V$.

The delocalized nature of the DNA excitonic states can also be illustrated by visualizing individual contributions to the total electric transition moment $\boldsymbol{\mu}_{E}=\sum c_{E i} \boldsymbol{\mu}_{i}=\sum_{i} \overline{\boldsymbol{\mu}}_{i}$. As an example, the mono-chromophoric contributions $\bar{\mu}_{i}$ are plotted as the red arrows in Figure 11 for selected vibrational states of the regular $\mathrm{d}(\mathrm{CG})_{72} * \mathrm{~d}(\mathrm{GC})_{72}$ polymer (top) as well as for the nucleosome particle DNA (bottom). In the delocalized $\mathrm{d}(\mathrm{CG})_{72} *$ $\mathrm{d}(\mathrm{GC})_{72}$ states 100 and 101 phases of the individual oscillators are synchronized along the entire helix. For the state number 6 which exhibits low spectral intensities the phases are synchronized within local nodes. In both cases the dipolar pattern reflects the helical symmetry of the system that is close to a rod. Within the applied model, the total transition dipole moment (the red line in Fig. 11) is always perpendicular to the helical axis, as only dipoles parallel to the base pair planes contribute to it. For the nucleosome (bottom part of Fig. 11) the periodic DNA structure is perturbed and does not allow for a large-scale excition delocalization. Nevertheless, the mode $2^{\prime}$, which is delocalized at least on a smaller scale, exhibits a notably higher $\mathrm{CD}$ $\left(R \sim 10^{-5}\right)$ than mode $73^{\prime}\left(R \sim 10^{-8}\right)$ localized practically on one chromophore only.

Further simulations with multiple DNA helices parallel to each other (not shown) indicate that the conclusions presented above are quite general and valid also for more complicated arrangements. For two parallel regular helices, for example, the CD-enhancement is only slightly perturbed for the interhelical distance of $25 \AA$, while at $50 \AA$ their interaction has no visible effect on the spectral shapes. For interhelical distances below 25 $\AA$, the CD enhancement decreases with the distance, and finally vanishes for very close helices. An increase of the number of the helices up to nine or variation of the transitional dipole frequency $\left(33,333 \mathrm{~cm}^{-1} \rightarrow 1680 \mathrm{~cm}^{-1}\right)$ did not bring any qualitative change. Similar observations were made also for various toroidal systems and multiple toroids.

\section{Conclusions}

CD spectra of DNA were simulated by extending the TDC scheme beyond the usual dipolar and magnetic dipolar/electric quadrupolar approximation. Instead, the field expansion was realized on a local level, in vicinity of individual chromophores. This enabled us to simulate the spectroscopic response for long DNA segments and other large model systems. We found that long-range phonon-like delocalized DNA excitations are most important for the enhancement of the CD signal. These simulations complement and extend the theory of the light interaction with large molecular systems previously proposed by Keller and coworkers, and are consistent with available experimental data. The TDC model appears suitable for prediction of the CD 

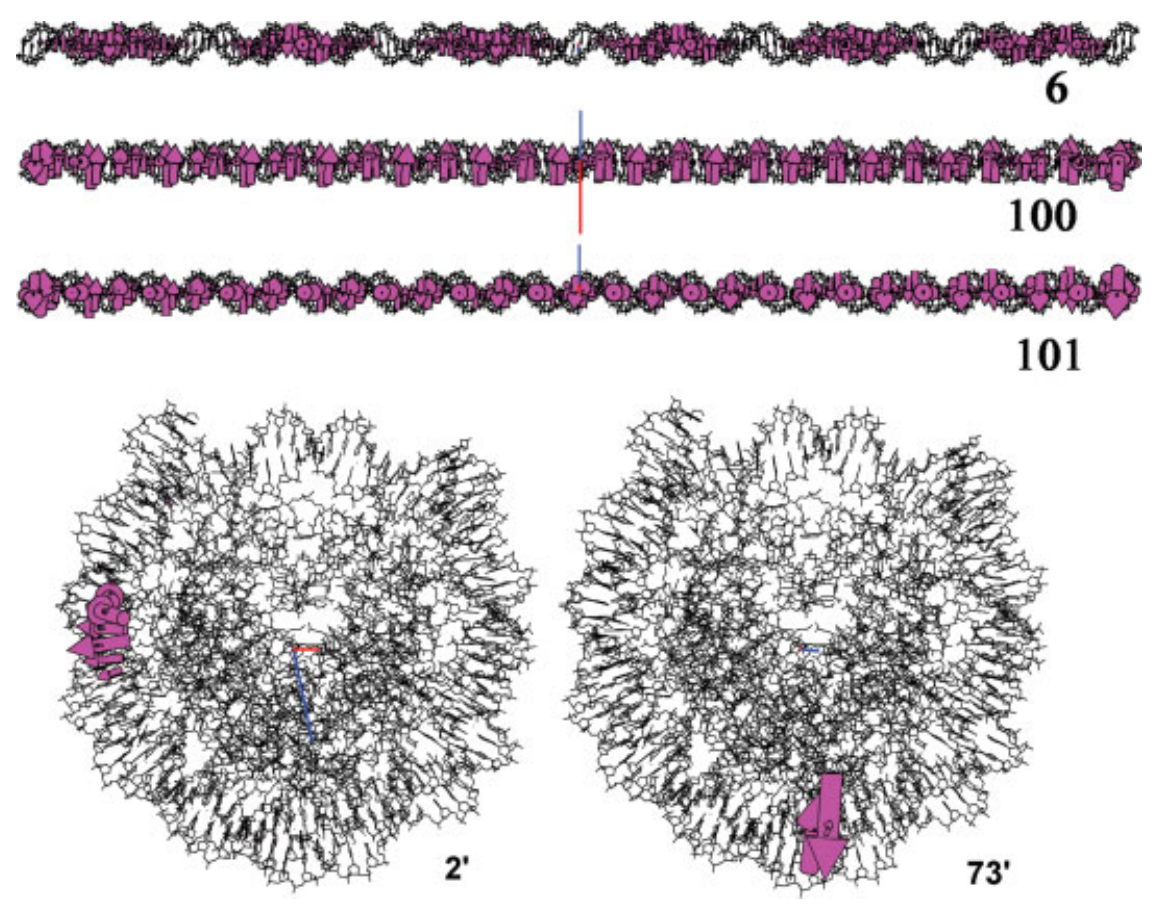

Figure 11. Visualization of the transition dipole contributions for selected TDC states: For the regular $\mathrm{d}(\mathrm{CG})_{72} * \mathrm{~d}(\mathrm{GC})_{72}$ DNA helix (top) state number 6 is not spectrally active, whereas states 100 and 101 provide large dipolar and rotational strengths. The nucleosome (bottom) does not favor CD enhancement. Nevertheless the partially delocalized mode $2^{\prime}$, for example, has significantly larger spectral signals than the localized state $73^{\prime}$. The blue and red lines indicate direction of the total transition magnetic and electric dipole moments, respectively. [Color figure can be viewed in the online issue, which is available at www.interscience.wiley.com.]

pattern of compact regular DNA systems where the spectral enhancement is favored by a mutual support of the nucleic acid threads. It appears that delocalized phonon-like DNA excitations can favor the $\psi$-CD effect in both electronic and vibrational spectral regions. The presented theoretical tools can thus be used for spectroscopic structural studies of large DNA systems.

\section{Acknowledgments}

The authors thank R. Pelc (The Stentor Institute) for the suggestions to the manuscript.

\section{References}

1. Bloomfield, V. A. Biopolymers 1991, 31, 1471.

2. Bloomfield, V. A. Biopolymers 1997, 44, 269.

3. Widom, J.; Baldwin, R. L. J Mol Biol 1980, 144, 431.

4. Shapiro, J. T.; Leng, M.; Felsenfeld, G. Biochemistry 1969, 8, 3219.

5. Khadake, J. R.; Rao, M. R. Biochemistry 1995, 34, 15792.

6. Mura, C. V.; Stollar, B. D. Biochemistry 1984, 23, 6147.

7. Liao, L. W.; Cole, R. D. J Biol Chem 1981, 256, 6751.

8. Cowman, M. K.; Fasman, G. D. Proc Natl Acad Sci USA 1978, 75, 4759.

9. Lerman, L. S. Proc Natl Acad Sci USA 1971, 68, 1886.
10. Jordan, C. F.; Lerman, L. S.; Venable, J. H. Nat New Biol 1972, 236, 67.

11. Evdokimov, Y. M.; Platonov, A. L.; Tikhonenko, A. S.; Varshavsky, Y. M. FEBS Lett 1972, 23, 180.

12. Evdokimov, Y. M.; Pyatigorskaya, T. L.; Kadikov, V. A.; Polyvtsev, O. F.; Doskocil, J.; Koudelka, J.; Varshavsky, Y. M. Nucleic Acids Res 1976, 3, 1533.

13. Gosule, L. C.; Schellman, J. A. Nature 1976, 259, 333.

14. Haynes, M.; Garrett, R. A.; Gratzer, W. B. Biochemistry 1970, 9 , 4410.

15. Zacharias, W.; Martin, J. C.; Wells, R. D. Biochemistry 1983, 22, 2398.

16. Gersanovski, D.; Colson, P.; Houssier, C.; Fredericq, E. Biochim Biophys Acta 1985, 824, 313.

17. Fasman, G. D.; Schaffhausen, B.; Goldsmith, L.; Adler, A. Biochemistry 1970, 9, 2814.

18. Andrushchenko, V.; Leonenko, Z.; Cramb, D.; van de Sande, H.; Wieser, H. Biopolymers 2001, 61, 243.

19. Maniatis, T.; Venable, J. H. J.; Lerman, L. S. J Mol Biol 1974, 84, 37.

20. Davey, C. A.; Sargent, D. F.; Luger, K.; Maeder, A. W.; Richmond, T. J. J Mol Biol 2002, 319, 1097.

21. Olins, D. E.; Olins, A. L. J Mol Biol 1971, 57, 437.

22. Cheng, S. M.; Mohr, S. C. FEBS Lett 1974, 49, 37.

23. Holzwarth, G.; Holzwarth, N. A. W. J Opt Soc Am 1973, 63, 324.

24. Keller, D.; Bustamante, C. J Chem Phys 1986, 84, 2961.

25. Keller, D.; Bustamante, C. J Chem Phys 1986, 84, 2972.

26. Kim, M. H.; Ulibarri, L.; Keller, D.; Maestre, M. F.; Bustamante, C. J Chem Phys 1986, 84, 2981. 
27. Evdokimov, Y. M.; Akimenko, N. M.; Glukhova, N. E.; Tikhonenko, A. S.; Varshavsky, Y. M. Mol Biol (USSR) 1972, 7, 124. (transl).

28. Craig, D. P.; Thirunamachandran, T. Molecular Quantum Electrodynamics; Dover Publications: New York, 1998.

29. Tinoco, I. Radiat Res 1963, 20133.

30. Bouř, P.; Keiderling, T. A. J Am Chem Soc 1992, 114, 9100.

31. Self, B. D.; Moore, D. S. Biophys J 1998, 74, 2249.

32. Maharaj, V.; Rauk, A.; van de Sande, J. H.; Wieser, H. J Mol Struct 1997, 408, 315.

33. DeVoe, H. J Phys Chem 1971, 75, 1509.

34. Zhong, W.; Gulotta, M.; Goss, D. J.; Diem, M. Biochemistry 1990, 29, 7485 .

35. Koslowski, A.; Sreerama, N.; Woody, R. W. In Circular Dichroism Principles and Applications; Nakanishi, K.; Berova, N.; Woody, R. W., Eds.; Wiley-VCH: New York, 2000, pp 97-132.

36. Chin, D. H.; Woody, R. W.; Rohl, C. A.; Baldwin, R. L. Proc Natl Acad Sci USA 2002, 11, 15416.

37. Rogers, D. M.; Besley, N. A.; O'shea, P.; Hirst, J. D. J Phys Chem B 2005, 109, 23061.

38. Rogers, D. M.; Hirst, D. M. Biochemistry 2004, 43, 11092.

39. Besley, N. A.; Hirst, J. D. J Am Chem Soc 1999, 121, 9636.

40. Barron, L. D. Molecular Light Scattering and Optical Activity; Cambridge University Press: Cambridge, 2004.

41. Charney, E. The Molecular Basis of Optical Activity; Wiley-Interscience: New York, 1979.

42. Andrews, D. L.; Thirunamachandran, T. J Chem Phys 1977, 67, 5026.

43. Zhong, W.; Gulotta, M.; Goss, D. J. Biochemistry 1990, 29, 7485.

44. Andrushchenko, V.; Wieser, H.; Bouř, P. J Phys Chem B 2003, 91, 12623.
45. Frisch, M. J.; Trucks, G. W.; Schlegel, H. B.; Scuseria, G. E.; Robb, M. A.; Cheeseman, J. R.; Montgomery, J. A., Jr.; Vreven, T.; Kudin, K. N.; Burant, J. C.; Millam, J. M.; Iyengar, S. S.; Tomasi, J.; Barone, V.; Mennucci, B.; Cossi, M.; Scalmani, G.; Rega, N.; Petersson, G. A.; Nakatsuji, H.; Hada, M.; Ehara, M.; Toyota, K.; Fukuda, R.; Hasegawa, J.; Ishida, M.; Nakajima, T.; Honda, Y.; Kitao, O.; Nakai, H.; Klene, M.; Li, X.; Knox, J. E.; Hratchian, H. P.; Cross, J. B.; Bakken, V.; Adamo, C.; Jaramillo, J.; Gomperts, R.; Stratmann, R. E.; Yazyev, O.; Austin, A. J.; Cammi, R.; Pomelli, C.; Ochterski, J. W.; Ayala, P. Y.; Morokuma, K.; Voth, G. A.; Salvador, P.; Dannenberg, J. J.; Zakrzewski, V. G.; Dapprich, S.; Daniels, A. D.; Strain, M. C.; Farkas, O.; Malick, D. K.; Rabuck, A. D.; Raghavachari, K.; Foresman, J. B.; Ortiz, J. V.; Cui, Q.; Baboul, A. G.; Clifford, S.; Cioslowski, J.; Stefanov, B. B.; Liu, G.; Liashenko, A.; Piskorz, P.; Komaromi, I.; Martin, R. L.; Fox, D. J.; Keith, T.; Al-Laham, M. A.; Peng, C. Y.; Nanayakkara, A.; Challacombe, M.; Gill, P. M. W.; Johnson, B.; Chen, W.; Wong, M. W.; Gonzalez, C.; Pople, J. A.; Gaussian, Inc.: Wallingford CT, 2004.

46. Becke, A. Phys Rev A 1988, 38, 3098.

47. Becke, A. D. J Chem Phys 1993, 98, 5648.

48. Jamorski, C.; Casida, M. E.; Salahub, D. R. J Chem Phys 1996, 104, 5134.

49. Varsano, D.; Garbesi, A.; DiFelice, R. J Phys Chem B 2007, 111, 14012.

50. Bayley, P. M. Prog Biophys Mol Biol 1973, 27, 1.

51. Tsankov, D.; Kalisch, B.; Van de Sande, J. H.; Wieser, H. Biopolymers 2003, 72, 490.

52. Tsankov, D.; Maharaj, V.; van de Sande, J. H.; Wieser, H. Vib Spectrosc 2007, 43, 152. 